\title{
The Tumor-Associated Macrophage-M2-Cancer Cell Complex and the Observation of Heterogeneous Modification of the Morphological Structure of Lung Adenocarcinoma
}

This article was published in the following Dove Press journal:

OncoTargets and Therapy

Wei-Hua Xiao ID
Li-Ping Yao
Min Li'
Min Wang'
Liang Wu'
Mao-Fen Jiang'
Hai-Fen Ma
Jun-Qiang Li'
Guo-Rong Chen ${ }^{2}$
'Department of Pathology, Ningbo Beilun
People's Hospital, Ningbo 3I5800,
People's Republic of China; ${ }^{2}$ Department
of Pathology, The First Affiliated Hospital
of Wenzhou Medical University,
Wenzhou 325000, People's Republic of
China

Correspondence: Wei-Hua Xiao

Department of Pathology, Ningbo Beilun People's Hospital, Ningbo 315800,

People's Republic of China

Tel +86057486100266

Fax +8605748677 6335

Email xiaoweihua_dr66@I63.com

Guo-Rong Chen

Department of Pathology, The First

Affiliated Hospital of Wenzhou Medical

University, Wenzhou 325000, People's

Republic of China

$\mathrm{Tel} / \mathrm{Fax}+86057786689908$

Email chengr_dr3366@163.com

\begin{abstract}
Objective: The aim of this study was to investigate the effect of the tumor-associated macrophage-m2-cancer cell complex (TAM-M2-CC) on the heterostructural modification of lung adenocarcinoma.
\end{abstract}

Methods: The expression of CD163+/CD68+ in macrophages in the microenvironment of 161 cases of lung adenocarcinoma was identified by dual immunohistochemistry, and the association between a TAM-M2-CC and its growth, as well as the histological changes in lung adenocarcinoma cells, was assessed.

Results: The morphological change of lung adenocarcinoma was related to the number of $\mathrm{m} 2$ phenotypes of the macrophages in the microenvironment of lung adenocarcinoma. TAM$\mathrm{M} 2-\mathrm{CCs}$ were involved in the process of cancer cell recognition, association, and reconstruction.

Conclusion: The microenvironment of lung adenocarcinoma can affect the phenotypic distinction of macrophages, and the polarization recruitment, zombification, and formation of a TAM-M2-CC, which can also affect the local differentiation of lung adenocarcinoma to a certain extent. The applicable pathogenesis needs to be verified and studied further.

Keywords: tumor-related macrophage-M2-cancer cell complex, TAM-M2-CC, heterostructural modification, microenvironment of lung adenocarcinoma, phenotypic differentiation, local differentiation

\section{Introduction}

Recently, it has been gradually acknowledged that the M2 phenotype of macrophages in the microenvironment of lung adenocarcinoma encourages the progress of lung adenocarcinoma and leads to a poor prognosis in patients. However, these studies have not shown whether the phenotypic cells are related to the morphological and structural changes of lung adenocarcinoma. In a previous study on macrophages, the researchers found that there were often many macrophages in the lung adenocarcinoma tissue, and these cells regularly gathered together in the infiltrating tumor. When some macrophages were closely mixed with free cancer cells, they easily formed a special structural complex; the tumor-associated macrophage-M2-cancer cell complex (TAM-M2-CC). This inadvertent finding has aroused our interest because there is no relevant literature regarding this complex, and its mechanism in the invasion and evolution of lung adenocarcinoma is still 
unknown. The following experiments were conducted with a view to discovering more about the relationship and significance of this complex to the structural heterogeneity of lung adenocarcinoma.

\section{Information and Methods}

\section{Research Materials}

The paraffined samples of 161 cases of lung adenocarcinoma were collected from the Department of Pathology in the First Affiliated Hospital of Wenzhou Medical University. All the cases were diagnosed from a doubleblinded approach, according to the classification standard of lung adenocarcinoma. ${ }^{1,2}$ Of the 161 lung cancer patients, 86 were male and 75 were female, and their ages ranged from 25 to 76 years (the average age was 58.3). No adjuvant chemotherapy or radiotherapy was completed in any of the cases before or during the surgery.

I confirm that I have read the Editorial Policy pages. This study was conducted with approval from the Ethics Committee of Ningbo Beilun People's Hospital. This study was conducted in accordance with the declaration of Helsinki. Written informed consent was obtained from all participants.

\section{The Main Reagents}

The main antibodies included CD68 (clone KP, mouse anti-human monoclonal antibody, trypsinized) and CD163 (clone 10D6, mouse anti-human monoclonal antibody, high-temperature repair), and they were all purchased from Fuzhou Maxim Biotechnology Co., Ltd. (China). The Dako EnVision ${ }^{\mathrm{tm}} \mathrm{G} 2$ Double-stain system (K5361150, for persons $\times 2$ ) used in this study consisted of the following: ready-to-use peroxygenase blocker; ready-to-use polymer/horseradish peroxidase (HRP), $33 \mathrm{~mL}$; diaminobenzidine (DAB) + substrate buffer, DAB + working solution; ready-to-use double staining blocking solution; Rabbit/Mouse (LINK); Permanent Red Substrate Buffer; Permanent Red Substrate Buffer solution; and Permanent Red Chromogen solution and water-based sealer.

\section{The Main Methods}

\section{The Double Immunohistochemical Staining} Procedure

After dewaxing and hydrating the paraffined samples, the remaining buffer was removed, and the samples were dried. A $200 \mu \mathrm{L}$ measure of the dual endogenous enzyme blocker was added to the sample, which was incubated for five minutes, and then washed for five minutes. Afterwards, a negative control group was prepared. After drying the buffer by shaking it, $200 \mu \mathrm{L}$ of the mouse antiCD163 was added. After incubation for 10 minutes, the samples were washed with a phosphate-buffered saline (PBS) and sliced into parts. A $200 \mu \mathrm{L}$ measure of polymer/HRP was then added to the parts, which were incubated for 10 minutes. After the sections were washed, a fresh DAB + working solution was prepared and $200 \mu \mathrm{L}$ of the $\mathrm{DAB}+$ working solution was added to them. The sections were incubated for eight minutes, washed clean with deionized water, and a double staining blocking agent was added, and they were incubated for three minutes. The parts were then washed, and the negative control reagent was added again. The buffer was removed, the best dilution ratio of mouse anti-CD68 was added, the sections were incubated for eight minutes, $200 \mu \mathrm{L}$ of the rabbit anti-rat cross-linking reagent was added, and they were incubated for a further 10 minutes. The excess flushing fluid was then removed by shaking the sections, and the polymer/AP was added and they were incubated again for 10 minutes. After this, the sections were washed twice, a temporarily prepared permanent red working solution was mixed with them and they were incubated for $15 \mathrm{~min}$ utes. Next, they were washed with distilled water, soaked in a glass cylinder with deionized water for five minutes, counter-stained with hematoxylin for three minutes, and then rinsed with distilled water for two to five minutes. Afterwards, the sections were sealed with the water-based sealing agent $\mathrm{C} 0563$, dried and sealed again with a neutral gum, and, finally, observed under a microscope.

\section{Cell Identification and Measurement}

The double staining showed that the first antibody contained brown granules and the second antibody included red granules. The cells with the two overlapping antibodies CD163+/CD68+ were dual expressing cells and had a bluish-purple color. These cells, which are easily observable and recognizable under a microscope, were acknowledged to be TAM-TC cells.

\section{The Complex Recognition, Counting, and Morphological Correlation Methods}

The binding of free cancer cells to the M2 phenotype of tumor-associated macrophages was identified, according to the localization of double immunomarkers in the lung adenocarcinoma. The firm and compact chimera consisting 
of more than two cancer cells and more than three macrophages were defined as a TAM-M2-CC, which manifested as a cord-like, small lump-like or mulberry-like structure in morphology. The association between the morphological changes of lung adenocarcinoma and the infiltration of the M2 macrophage was observed under a microscope and the total number of TAM-M2-CCs in five 200-time fields was observed and calculated using the double-blind method. The mean value of the two counts was considered to be the result.

\section{Experimental Grouping}

The experimental lung adenocarcinoma group was divided into three groups: The in situ (AIS), micro-invasive (MIA), and invasive (IA) groups, and a squamous carcinoma positive control group was also established. As there was no tumor-associated macrophage-M2-cancer cell complex (TAM-M2-CC) in the normal lung tissue adjacent to the tumor, no negative control was established. The association between TAM-M2-CCs and the histology of different lung adenocarcinomas was observed. There were four morphological association groups: The simple adenoid group, irregular adenoid group, complex adenoid group (with a sieve or labyrinth-like structure), and the solid nest-like group.

\section{Statistical Analysis}

IBM SPSS 24.0 Statistics and GraphPad Prism 8.3.0 were used for the data and chart analysis. The comparison between the two groups was completed using a one-way analysis of variance (ANOVA), or nonparametric KruskalWallis test, and two samples in the same group were compared using an unpaired/independent two-tailed $t$-test. The data were first tested for the homogeneity of variance using an F-test, and then evaluated using a $t$-test. If an F-test revealed a value of $\mathrm{P}<0.05$, a Welch correction $t$-test was used. Whether the actual data was normally distributed was determined according to the QQ-plot diagram, and non-normally distributed data were compared using a nonparametric Mann-Whitney $U$-test. $\mathrm{P}<0.05$ was considered statistically significant.

\section{Results}

\section{The Morphological Observation of TAM- M2 Immune Expression}

TAM-M2 cells could be identified in the lung adenocarcinoma tissues, mainly in the inner and marginal areas of the cancer tissues. Under a light microscope, the TAM-M2 cells were brown and co-expressed both CD163 and
CD68. The double-stained particles were located in the cytoplasm and the membrane of the macrophages. The cell morphology varied and the cell sizes changed from small, medium, to large. Most of the cells were mononuclear, and a few of the cells merged into multinuclear giant cells. In the tumor-associated macrophage-M2-cancer cell complex (TAM-M2-CC), which was comprised of macrophages and endo-genetic budding cancer cells, the TAM-M2 cells had a more varied morphology, a rich cytoplasm, and were round, oval or irregularly elongated. A few of the macrophages close to the cancer cells showed protrusions, which adhered to the protrusions formed by the cytoplasm on the surface of the cancer cells. Certain special structures could be seen in some samples, such as the inclusion body, multipole, and cliff suspension bridge. Occasionally, transmural, reticular, complex, giant or syncytia structures could be seen (Figure 1).

\section{The Histological Correlation of Tumor- Associated Macrophage-M2-Cancer Cell Complexes (TAM-M2-CCs) with the Morphological Heterogeneity of Different Lung Adenocarcinomas}

Tumor-associated macrophages are commonly found in AIS, adherent MIA, and adherent IA types of lung adenocarcinoma, but TAM-M2-CCs associated with cancer epithelial cells are rare. In adenocarcinomas with an irregular adenoid structure, there are scattered linear folds, in which there are a few TAM-M2-CCs, but there is a tendency of the existing M2 macrophages to gather at the tip of the irregular folds or around the bottom of the adjacent folds, presenting an unstable connection state. In the complex adenoid group, the spatial structure of M2 macrophage aggregation was more complex, the degree of the three-dimensional overlap was enhanced, and the size of the complex was diverse. The chance of contact between the complex and intraluminal surface of cancer was increased because the root could adhere to the budding part of the cancer cells, where the expression of antigen on the surface of the cancer cells was the same as that of macrophages, and the positive degree was weakened and limited. The TAM-M2-CC cells were mosaic-like with the cancer cells, establishing a three-dimensional structure of a snowflake or a twist-like structure of TAM-M2-CCs, and they grew in a spiral of weaving or knitting. The long axis of the complex was at an angle to the basal surface of the internal cavity of the cancer cells, and there were multiple 

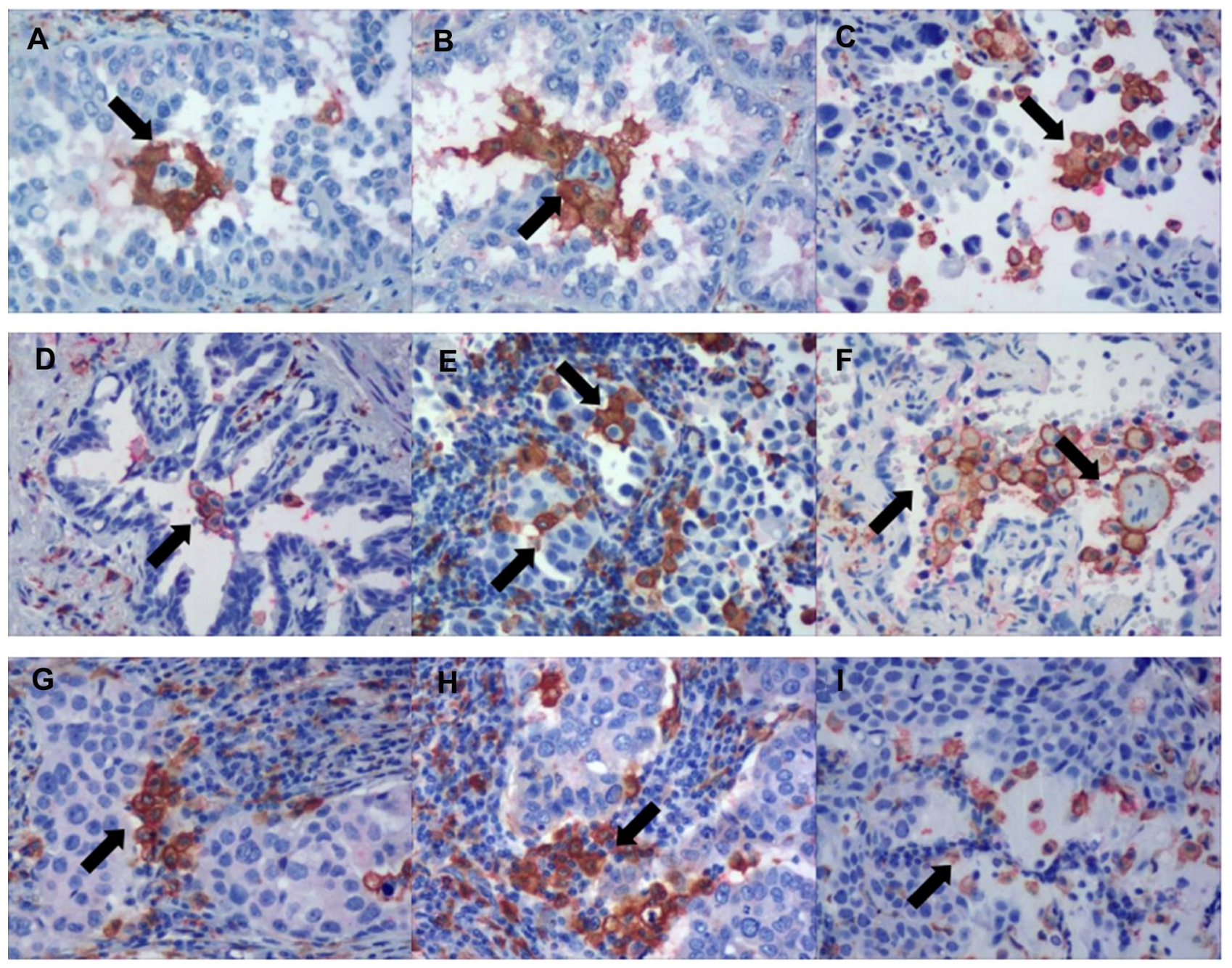

Figure I The different morphological manifestations of a TAM- M2-CC. (A) Partial or complete inclusion body structure. (B) Radial inclusion body structure. (C) The end extends and then sticks out. (D) Adjacent nipple top cliff suspension bridge like connection. (E) Segmented and sutured malignant gonads. (F) A synzygotic structure. (G) Vertical "welding" between cancer nests. (H) A lateral cement sample between nests. (I) Lymphocyte bridge. The TAM-M2-CC is indicated (see arrows).

cord-like structures, which fused with ease. The average fusion rate was $43.5 \%$. In the well-formed cancer nests, the number of macrophages and complexes decreased or disappeared. In the solid nests, the TAM-M2-CCs were significantly reduced and only existed in the focus area of a few samples. There were few individual TAM-M2 cells in the stroma around the cancer nests; TAM-M2-CCs were uncommon in this area (Figure 2).

\section{The Infiltration Role of Tumor-Associated Macrophage-M2-Cancer Cell Complexes (TAM-M2-CCs) in the Experimental}

\section{Groups and the Control Group}

A Kruskal-Wallis test was performed to make an inter-group comparison of the changing quantities of the TAM-M2-CC, using SPSS 24.0 software and GraphPad prism 8.3.0. The results showed that there was a significant difference in the number of TAM-M2-CCs between the experimental groups and the control group $(\mathrm{z}=120.20, \mathrm{P}<0.001)$. The data of two of the experimental groups were compared using the MannWhitney test. The number of TAM-M2-CCs in the AIS group was significantly less than that in the IA group ( $\mathrm{U}=1140.00$, $\mathrm{P}<0.001$ ). There was no significant difference in the number of TAM-M2-CCs between the MIA group and the IA group $(\mathrm{U}=659.00, \mathrm{P}=0.814)$. The comparison between the MIA group and the IA group showed that TAM-M2-CCs were normally distributed, and after completing the Welch correction and the evaluation using a $t$-test, there was seen to be a significant difference between the two groups $(\mathrm{t}=6.11$, $\mathrm{P}<0.001)$. The number of TAM-M2-CCs in the AIS, MIA, and IA groups were also significantly different from the 

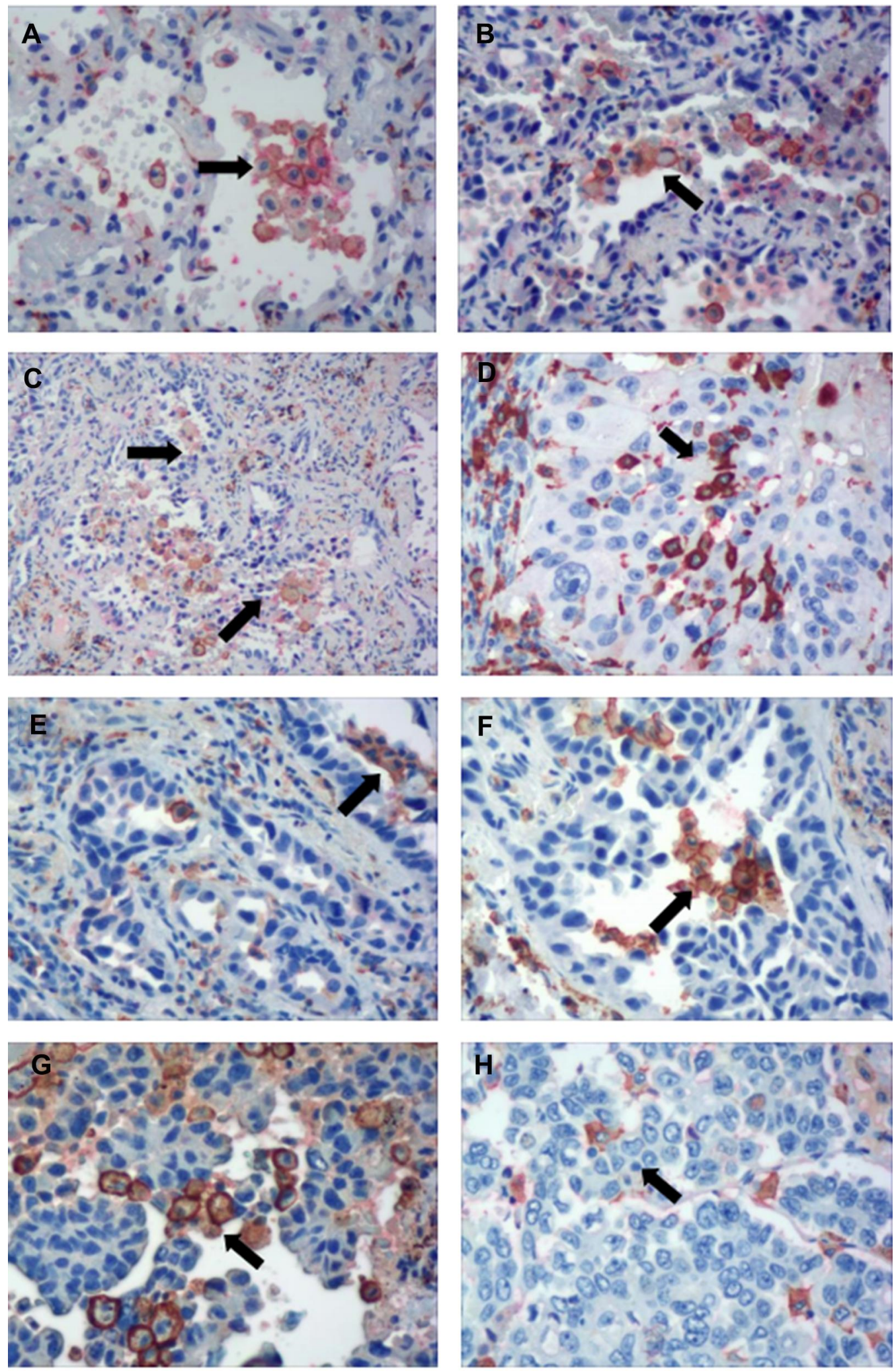

Figure 2 Histological correlation of TAM-M2-CC with morphologic heterogeneity of different lung adenocarcinoma by the double Immunohistochemical staining. 
Table I Distribution of TAM-M2-CC in Different Lung Adenocarcinoma

\begin{tabular}{|l|l|l|}
\hline Groups & Cases & TAM-M2-CC(Apiece/5×200 Times) \\
\hline Control & 50 & $3.68 \pm 1.81$ \\
AIS & 67 & $8.92 \pm 4.60$ \\
MIA & 18 & $23.14 \pm 9.58$ \\
IA & 76 & $23.72 \pm 15.68$ \\
LA & 161 & $17.50 \pm 13.66$ \\
\hline$Z$ Value & & 120.20 \\
$P$ value & & $P<0.0001$ \\
\hline
\end{tabular}

Notes: The number of TAM-M2-CC is expressed as (mean \pm SD); Single-factor Kruskal-Wallis statistic was used for comparison among the five groups; $P<0.05$ was statistically significant.

Abbreviations: AIS, lung adenocarcinoma in situ; MIA, tiny invasive adenocarcinoma; IA, invasive adenocarcinoma; LA, total lung adenocarcinoma.

number in the LA group (the statistical values were $\mathrm{U}_{\mathrm{AIS} / \mathrm{LA}}=$ 3476.00 and $\mathrm{P}<0.001, \mathrm{U}_{\mathrm{MIA} / \mathrm{LA}}=912.50$ and $\mathrm{P}=0.05$ and $\mathrm{U}_{\mathrm{IA} / \mathrm{LA}}=4737.00$ and $\mathrm{P}<0.05$ ), (Table 1, Figure 3).

\section{The Relationship Between the Number of} TAM-M2-CCs and the Morphological Heterogeneity of Lung Adenocarcinoma

TAM-M2-CCs had varied individual or cluster morphologies in different forms of lung adenocarcinoma. The research team focused on assessing the changes in quantity and the patterns of change in the TAM-M2-CCs in the different morphological association groups as well as in the control group. The difference in the changes in the number of TAM-M2-CCs among the groups was statistically significant ( $\mathrm{z}=144.70, \mathrm{P}<0.0001$ ). They were significantly elevated in the simple adenoid group, irregular adenoid group, complex adenoid group, and solid nest group, when compared to the control group. In the first three experimental groups, the number of TAM-M2-CCs was significantly increased, whereas it was significantly decreased in the last group although the number was still higher than in the first group. The number of TAM-M2$\mathrm{CCs}$ in the irregular adenoid group and the complex adenoid group differed $(\mathrm{t}=2.55, \mathrm{P}<0.05)$ (Table 2, Figure 4).

\section{The Observation of Other Components of TAM-M2-CCs}

In this study, we found that the M2 macrophage-cancer cell complex is comprised of more than two components, and a few cells were hyperchromatic and had bare nuclei, with a small amount of cytoplasm, similar to lymphocytes, signifying that the formation of the complex has a complex mechanism and other components, such as lymphocytes,
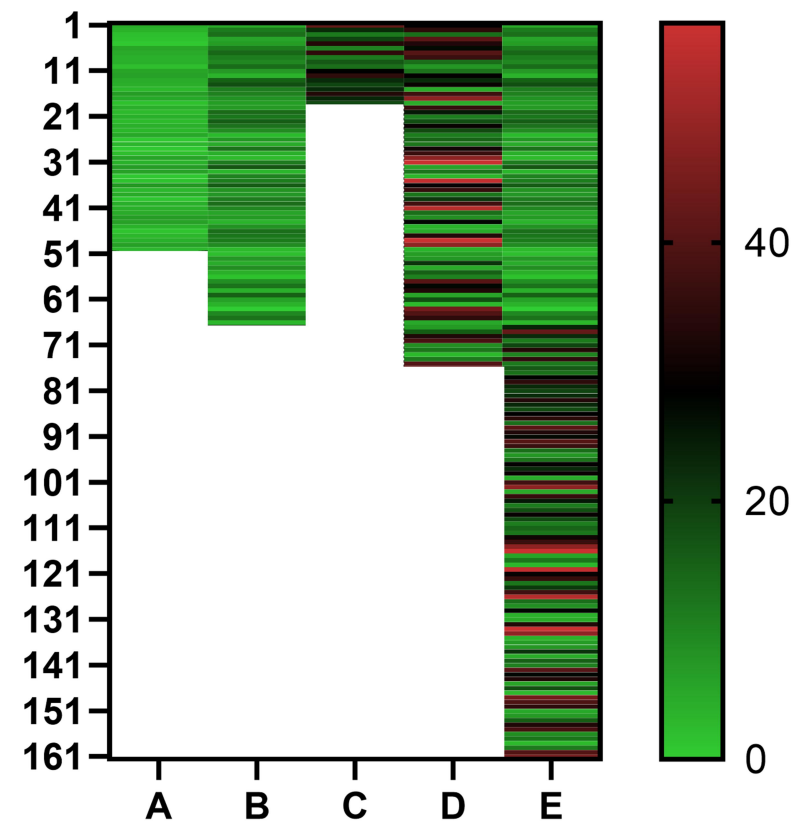

TAMs-M2-CC in Type groups

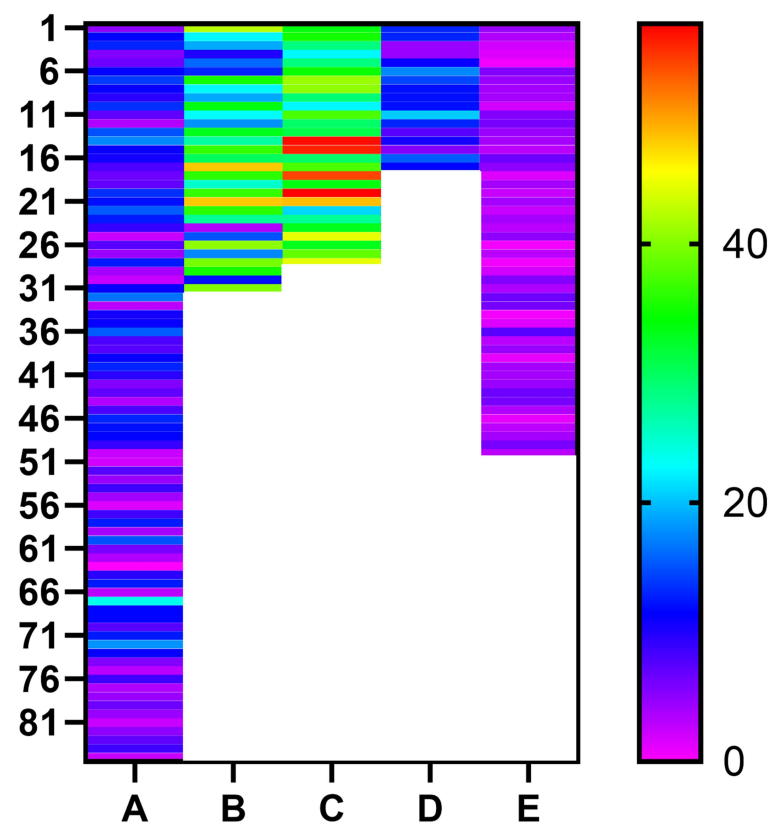

TAMs-M2-CC in Associated groups

Figure 3 The distribution pattern of hot spots in different lung adenocarcinoma and morphological association groups of TAM-M2-CCs. The left side: $A=$ control $B=A I S$ $C=M I A \quad D=I A E=L A$. The right side: $A=$ pure adenoid $B=$ irregular adenoid $C=$ complex adenoid $D=$ solid nests $E=$ control. 
Table 2 The Relationship Between the Number of TAM-M2-CC and the Morphological Heterogeneity of Lung Adenocarcinoma

\begin{tabular}{|l|l|l|}
\hline Groups & Cases & $\begin{array}{l}\text { TAM-M2-CC(Apiecel } \\
\mathbf{5 \times 2 0 0 ~ T i m e s ) ~}\end{array}$ \\
\hline Control & 50 & $3.68 \pm 1.81$ \\
Pure adenoid & 85 & $8.60 \pm 4.53$ \\
Irregular adenoid & 31 & $27.76 \pm 11.63$ \\
Complex adenoids & 28 & $36.70 \pm 10.32$ \\
Solid nests & 17 & $11.65 \pm 4.33$ \\
\hline Z Value & & 144.70 \\
P value & & P<0.000I \\
\hline
\end{tabular}

Notes: The number of TAM-M2-CC is expressed as (mean \pm SD); Single-factor Kruskal-Wallis statistic was used for comparison among the five groups; $\mathrm{P}<0.05$ was statistically significant.

may play a role in signal regulation. In certain areas, dense fine lymphocyte lines could be seen dividing the cancer glandular cavity, but there were no clear residual cancer cells and no TAM-M2 cells with a double positive expression of CD163+/CD68+. This suggests that this lymphocyte bridge may be a special stage of a TAM-M2-CC or another auxiliary growth mode (Figure 1I).

\section{Discussion}

Researchers have gradually recognized that distinctive properties of macrophages can be activated in different tumor microenvironments, and macrophages transform into phenotypes with diverse functional characteristics. Previous studies have revealed that the infiltration of a large number of macrophages is evident in the most invasive tumors since there were two different macrophage phenotypes in non-small cell lung cancer (NSCLC) (lung adenocarcinoma is one of them): M1 and M2 macrophages. ${ }^{3,4}$ The M2 phenotype of macrophages is oncophilic and encourages tumor invasion, with CD163 being a marker. The researchers' previous study also showed that the increase in M2 phenotypes of macrophages in lung adenocarcinomas is higher than the surrounding normal lung tissue far away from the tumor, and the M2 phenotypes of macrophages in the tumor parenchyma gather in a polar manner, which usually indicates a poor prognosis. ${ }^{5}$ Recently, researchers have also observed that the M2 phenotype of macrophages may be involved in the morphological and structural transformation of lung adenocarcinoma and the local alteration of the microenvironment. This phenomenon has not been previously reported in the literature at home or abroad.
In the present study, the M2 phenotype of macrophages in lung adenocarcinoma was labeled by double immunohistochemistry, and the role of changes of TAM-M2-CCs in lung adenocarcinoma was assessed under a microscope to preliminarily explain the association between M2 macrophages and the internal modification of lung gland morphology. These results were interesting since the preliminary morphological observation showed that TAMM2-CCs played a particular role in the differentiation and morphological heterogeneity of lung adenocarcinoma.

The results of the present study showed that the numbers of M2 phenotypes of macrophages were higher in lung adenocarcinoma than in the normal control group. However, there were some differences in the incidence of TAM-M2-CC in tumors and the complexity of structural areas. This was established to be the process of the complex beginning to form, adhere, and bridge, undergoing three-dimensional fusion, and then gradually degrading and disappearing. This phenomenon may be related to the immature condition of TAM-M2-CC formation in adenocarcinomas in adherent morphology. When the carcinomatous glands show a cribriform structure, the irregular changes in the primary gonadal cavity start the formation of the complex, accelerating the internal shaping and structural modification of the cancer focus. The complex is more likely to appear in different shapes of a sieve-like or solid structure. Also, the threshold of the number of molding TAM-M2-CCs in lung adenocarcinomas will increase after full solid transformation, and the complex will gradually disintegrate and disappear. The mechanism of this phenomenon may be the increase in the relevant signal factors formed by the contact inhibition of the cancer cells in the hypoxic microenvironment. ${ }^{6,7}$ Within the closed three-dimensional anoxic environment of cancer nests, the alteration of macrophages at the center of the cancer nests gradually becomes weaker and may even stop. As the macrophages in the cancer nests are surrounded by cancer cells, apoptosis increases, and nutrition becomes insufficient. A poor microenvironment also makes the macrophages decrease gradually until most, or all of them, are killed, and the obvious decrease or disappearance of macrophages makes the external transformation or modification of cancer nests relatively static.

The results of the present study show that macrophages play a role similar to worker ants. The change in the cancer focus was like the construction of an ant nest, from a single to a complex mechanism, and the macrophages, though simple adherent and acinar structures, 


\section{Type groups}

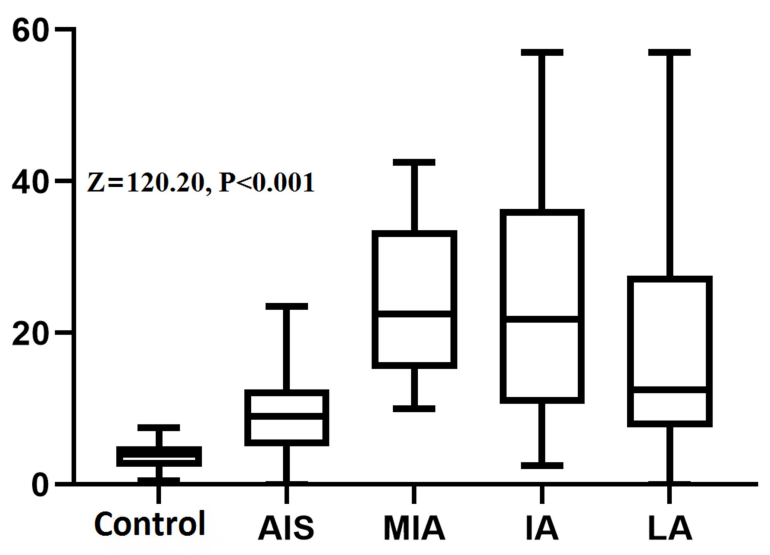

Type groups

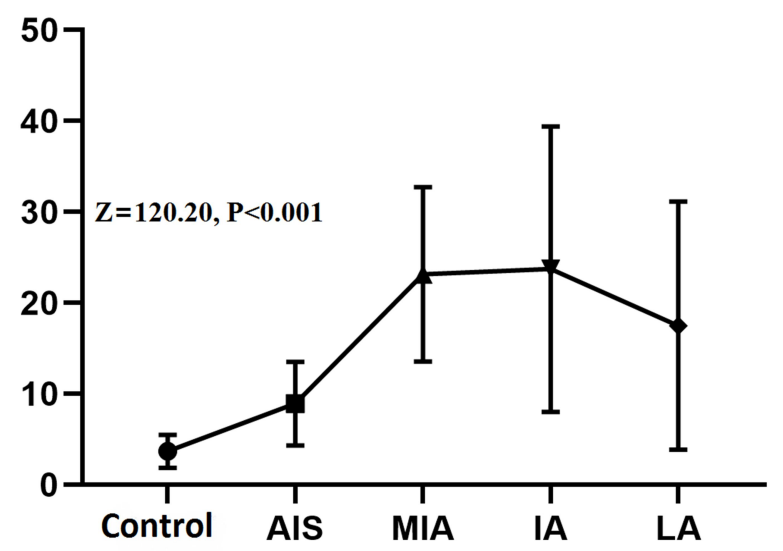

\section{Associated groups}

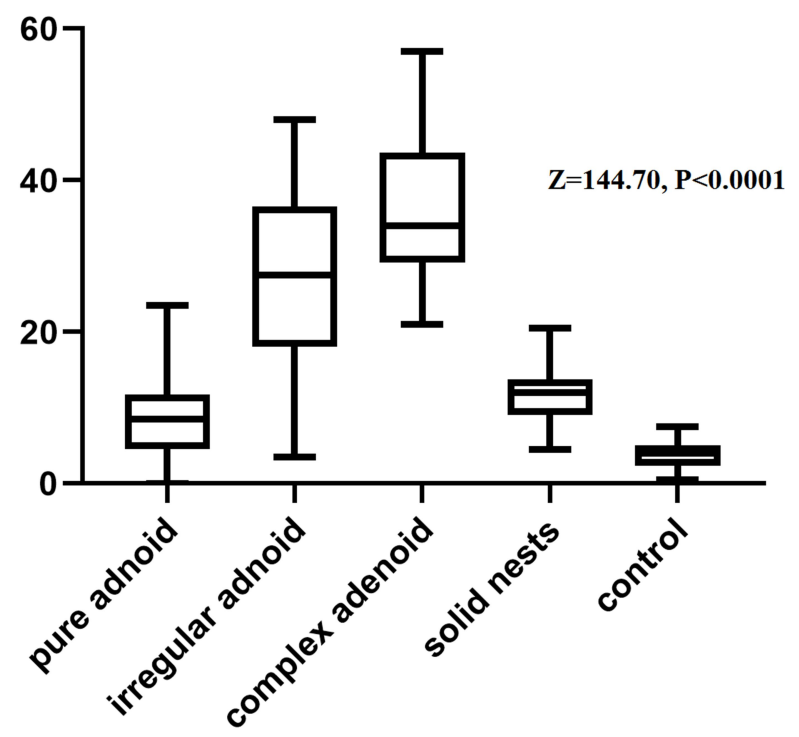

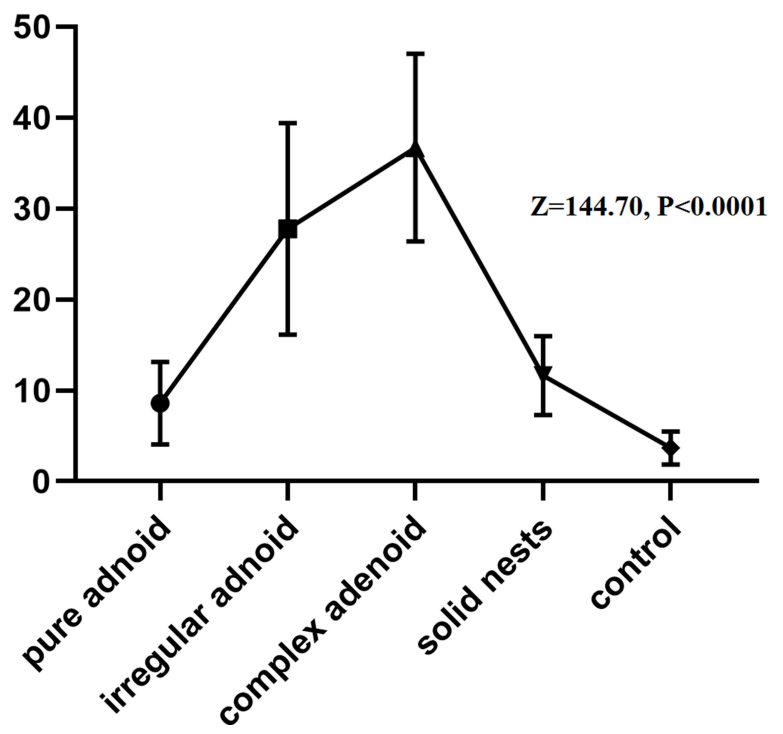

Figure $4 \mathrm{~A}$ comparison of the quantity of TAM-M2-CCs in the experimental group and in the morphological association group. Abbreviations: AIS, lung adenocarcinoma in situ; MIA, tiny invasive adenocarcinoma; IA, invasive adenocarcinoma; LA, total lung adenocarcinoma; Control group, squamous cell carcinoma.

could not easily form complexes with the cancer cells. The change in morphology was relatively simple, and this was the reason why the irregular glandular cavity was mainly associated with the degree of proliferation of the cancer cells. ${ }^{8,9}$ In some samples, we observed that TAM-M2-CCs were involved in the process of internal division and the connection or renovation of carcinomatous glands. The present study also showed that the appearance of an insect bite-like modification, transformation, and fibrosis of the macrophages around the cancer focus induced subtle changes in the direction and shape of the cancer cells infiltrating outwards. In terms of the growth model, the expansion and irregular invasive growth of the cancer cells alternated or occurred at the same time. Regarding limited growth space, the epithelial-mesenchymal transition occurred repeatedly and the fibrosis was visible. ${ }^{10,11}$ The macrophages frequently participated in the transformation of interstices around the cancer nests, which indirectly 
affected the marginal morphology of cancer to a certain extent. The number of macrophages consumed by these transformative activities was moderately large and the appearance of some immune tolerance signals may also have promoted the gradual disappearance of the M2 phenotype of macrophages. This phenomenon has been verified in the study of extrapulmonary diseases such as pancreatic ductal adenocarcinoma and the immunotherapy of other tumors. ${ }^{12,13}$

The general hypothesis is that, at first, cancer cells secrete many bait factors and a large number of tumor-associated macrophages are recruited to gather around the carcinomatous gonadal cavity. In the beginning, macrophages and other inflammatory cells jointly participate in the immune suppression of the cancer tissues. ${ }^{14-16}$ Macrophages initiate inflammatory phagocytosis at the weak points of the carcinomatous glands, and certain cancer cells are cut out. After a gap appears, the macrophages in the anastomotic cavity are entrapped and enriched, and the carcinomatous glands continue to enlarge and divide, or fill in the gap. Some M2 phenotype macrophages are temporarily pseudo-assimilated after coming into contact with the cancer cells, and a large number of mutinous homogenous epithelioid tumor-associated macrophages in the carcinomatous gonadal cavity are progressively replaced. These macrophages exhibit heterogeneous vacuolation in the cytoplasm, immune recognition, antigen presentation, and the phagocytic function is partially lost. ${ }^{17,18}$ For cancer cells, these macrophages are cultured zombie cells, which are a high-quality carrier for the viral colonization of cancer cells. The polarization of the M2 phenotype of macrophages may be associated with the decrease of some receptors, such as colony-stimulating factor-1 (CSF-1), on the cell surface, and the macrophage polarization is regulated by the applicable signaling pathway, such as the AMPK-NF- $\mathrm{KB}$ signaling pathway. ${ }^{19,20}$

Cancer cells begin a new round of proliferation and migration and effect adherent migration and metastasis with a plasmid construction around the adjacent macrophages, while the macrophages realize cancer cell structure shaping and heterogeneous programming. The cancer cells and macrophages then jointly form a chain sandwich complex. With the macrophage abscission, a new cancer cell bridge is formed under the action of protein adhesion. The above-mentioned process repeats itself, and the cancer cells undergo a transformation of their three-dimensional histological reticular structure, following a principle similar to DNA replication. Accordingly, the proliferate cancer cells form further bridges to fill the residual space and form solid structures. In the process of partaking in the structural programming and transformation of cancer cells, the number of macrophages and the direction of their arrangement has some impact on the new spatial structure of the cancer cells. The mode, direction, and degree of migration of the cancer cells around the macrophages also have a noticeable impact on the final shape of the transformed cancer nests. Further reasoning, based on the above-mentioned assumptions, suggests that with regard to the morphological heterogeneity of cancer cells, in addition to the gene and protein expression and other innate differentiation factors, macrophages may also play an important role in the secondary differentiation of cancer cells and the progression of the disease. Therefore, the above process reveals that cancer cells have a certain sociality, that is, they are the so-called "smart cells". The cancer cells simply use the macrophages for this process since tumor-associated macrophages are good carriers for cancer cells to complete the process of the proliferation, invasion, and evolution of the whole body.

Although activated macrophages are effector cells that phagocytize microorganisms and kill tumor cells, in the microenvironment induced by lung adenocarcinoma, the host immune response is inhibited, and the M2 phenotype of macrophages partakes in the activity of promoting tumor progression. ${ }^{21}$ In addition to the zombification and impotence of the M2 phenotype of macrophages, the formation of the complex may be the most critical factor in this process, even if the transformation effect of the complex on the microenvironment may be limited in the current data, and it often occurs in the moderately and poorly differentiated stage. Since the number of complexes in solid poorly differentiated adenocarcinoma is small, the reduction in the number of complexes may suggest a poor prognosis. The results of the present study indicate that there may be an inseparable association between the two, but the stage difference of the cancer tissue progression is also an important factor that cannot be ignored. In future clinical treatment, patients with poorly differentiated lung adenocarcinoma should first be treated with M2 macrophage reduction to reduce TAM-M2-CC formation and inhibit the malignant morphological transformation process of the cancer cells. This may help improve the prognosis of patients with lung adenocarcinoma. ${ }^{22}$

There are several limitations to this study. Firstly, the number of TAM-M2-CCs was calculated in two-dimensional tissue sections, which only partially described the true threedimensional architecture of these cells in-vivo. Secondly, 
early AIS/MIA tumors tend to be smaller than invasive adenocarcinomas, but tumor size was not controlled in the study. Lastly, the prognostic/pathologic significance of the changes in the number of TAM-M2-CCs was not investigated.

\section{Conclusion}

It is evident that the body's tolerance to tumor invasion can be enhanced through the corresponding effective heterogeneous morphological regulation and control of lung adenocarcinomas. However, the variety of complex components presents a difficulty for future research. The current literature available cannot prove the exact function and immune mechanism of lymphocytes in tumor-associated macrophage-m2-cancer cell complex (TAM-M2-CC) formation. However, a previous study has shown that there were some phased research results23 in studies on immune signal regulation, which interestingly referred to the initiation and braking mechanism of the complex, and this subject needs to be addressed further.

\section{Acknowledgments}

We are particularly grateful to all the people who have given us help on our article.

\section{Funding}

This study was funded by the Natural Project of the Ningbo Science and Technology Bureau (NO.2015A610231). The funding body had no role in the design of the study and collection, analysis, and interpretation of data and in writing the manuscript.

\section{Disclosure}

The authors declare that they have no competing interests.

\section{References}

1. Martinez FO, Helming L, Gordon S. Alternative activation of macrophages: an immunologic functional perspective. Annu Rev Immunol. 2009;27:451-483. doi:10.1146/annurev.immunol.021908.132532

2. Travis WD, Brambilla E, Noguchi M, et al. International association for the study of lung cancer/american thoracic society/european respiratory society international multidisciplinary classification of lung adenocarcinoma. J Thorac Oncol. 2011;6:244-285. doi:10.1097/ JTO.0b013e318206a221

3. Siveen KS, Kuttan G. Role of macrophages in tumour progression. Immunol Lett. 2009;123:97-102. doi:10.1016/j.imlet.2009.02.011

4. Ohri CM, Shikotra A, Green RH, Waller DA, Bradding P. Macrophages within NSCLC tumour islets are predominantly of a cytotoxic M1 phenotype associated with extended survival. Eur Respir J. 2009;33:118-126. doi:10.1183/09031936.00065708

5. Xiao WH, Zhao LL, Ying LL, et al. Polarized distribution of M2 macrophages in marginal region around lung adenocarcinoma and its effect on prognosis. Chin J Pathophysiol. 2015;31:160-165.
6. Cho WC, Kwan CK, Yau S, So PP, Poon PC, Au JS. The role of inflammation in the pathogenesis of lung cancer. Expert Opin Ther Targets. 2011;15:1127-1137. doi:10.1517/14728222.2011.599801

7. Solinas G, Germano G, Mantovani A, Allavena P. Tumor-associated macrophages (TAM) as major players of the cancer-related inflammation. J Leukoc Biol. 2009;86:1065-1073. doi:10.1189/jlb.0609385

8. Lo Sicco C, Reverberi D, Balbi C, et al. Mesenchymal stem cellderived extracellular vesicles as mediators of anti-inflammatory effects: endorsement of macrophage polarization. Stem Cells Transl Med. 2017;6:1018-1028. doi:10.1002/sctm.16-0363

9. Covarrubias AJ, Aksoylar HI, Yu J, et al. Akt-mTORC1 signaling regulates Acly to integrate metabolic input to control of macrophage activation. Elife. 2016;5:e11612. doi:10.7554/eLife.11612

10. Mitsuhashi A, Goto H, Kuramoto T, et al. Surfactant protein A suppresses lung cancer progression by regulating the polarization of tumor-associated macrophages. Am J Pathol. 2013;182:1843-1853. doi:10.1016/j.ajpath.2013.01.030

11. Yu CC, Chien CT, Chang TC. M2 macrophage polarization modulates epithelial-mesenchymal transition in cisplatin-induced tubulointerstitial fibrosis. Biomedicine (Taipei). 2016;6:5. doi:10.7603/ s40681-016-0005-5

12. An M, Li D, Yuan M, Li Q, Zhang L, Wang G. Interaction of macrophages and endometrial cells induces epithelial-mesenchymal transition-like processes in adenomyosis. Biol Reprod. 2017;96:46-57.

13. Guttman O, Freixo-Lima GS, Kaner Z, Lior Y, Rider P, Lewis EC. Context-specific and immune cell-dependent antitumor activities of $\alpha 1$-antitrypsin. Front Immunol. 2016;7:559. doi:10.3389/ fimmu.2016.00559

14. Szebeni GJ, Vizler C, Kitajka K, Puskas LG. Inflammation and cancer: extra- and intracellular determinants of tumor-associated macrophages as tumor promoters. Mediators Inflamm. 2017;2017:9294018. doi:10.1155/2017/9294018

15. Liu Y, Tu L, Wang L, et al. The accumulation of macrophages attenuates the effect of recombinant human endostatin on lung cancer. Onco Targets Ther. 2016;9:6581-6595. doi:10.2147/OTT.S114389

16. Iwanowycz S, Wang J, Hodge J, Wang Y, Yu F, Fan D. Emodin inhibits breast cancer growth by blocking the tumor-promoting feedforward loop between cancer cells and macrophages. Mol Cancer Ther. 2016;15:1931-1942. doi:10.1158/1535-7163.MCT-15-0987

17. Sok MCP, Tria MC, Olingy CE, San Emeterio CL, Botchwey EA. Aspirin-triggered resolvin D1-modified materials promote the accumulation of pro-regenerative immune cell subsets and enhance vascular remodeling. Acta Biomater. 2017;53:109-122. doi:10.1016/j. actbio.2017.02.020

18. Montenegro-Burke JR, Sutton JA, Rogers LM, Milne GL, McLean JA, Aronoff DM. Lipid profiling of polarized human monocytederived macrophages. Prostaglandins Other Lipid Mediat. 2016;127:1-8. doi:10.1016/j.prostaglandins.2016.11.002

19. Cypher LR, Bielecki TA, Huang L, et al. CSF-1 receptor signalling is governed by pre-requisite EHD1 mediated receptor display on the macrophage cell surface. Cell Signal. 2016;28:1325-1335. doi:10.1016/j.cellsig.2016.05.013

20. Chiang CF, Chao TT, Su YF, et al. Metformin-treated cancer cells modulate macrophage polarization through AMPK-NF- $\mathrm{BB}$ signaling. Oncotarget. 2017;8:20706-20718. doi:10.18632/oncotarget.14982

21. Chai CY, Chen WT, Hung WC, et al. Hypoxia-inducible factor1alpha expression correlates with focal macrophage infiltration, angiogenesis and unfavourable prognosis in urothelial carcinoma. $J$ Clin Pathol. 2008;61:658-664. doi:10.1136/jcp.2007.050666

22. Goswami KK, Ghosh T, Ghosh S, et al. Tumor promoting role of anti-tumor macrophages in tumor microenvironment. Cell Immunol. 2017;316:1-10. doi:10.1016/j.cellimm.2017.04.005

23. Zhou J, Qu Z, Sun F, et al. Myeloid STAT3 promotes lung tumorigenesis by transforming tumor immunosurveillance into tumor-promoting inflammation. Cancer Immunol Res. 2017;5:257-268. doi:10.1158/2326-6066.CIR-16-0073 


\section{Publish your work in this journal}

OncoTargets and Therapy is an international, peer-reviewed, open access journal focusing on the pathological basis of all cancers, potential targets for therapy and treatment protocols employed to improve the management of cancer patients. The journal also focuses on the impact of management programs and new therapeutic

Submit your manuscript here: https://www.dovepress.com/oncotargets-and-therapy-journal agents and protocols on patient perspectives such as quality of life, adherence and satisfaction. The manuscript management system is completely online and includes a very quick and fair peer-review system, which is all easy to use. Visit http://www.dovepress.com/ testimonials.php to read real quotes from published authors. 